\title{
Simple and compact Hz-level linewidth Laser System via improved mounting configuration of a Reference Cavity
}

\author{
Mark Notcutt, John L. Hall, Andrew Ludlow and Jun Ye \\ JILA, National Institute of Standards and Technology and University of Colorado, Boulder, Colorado 80309-0440. \\ Mark.Notcutt@Colorado.edu \\ Long-Sheng Ma \\ JILA, National Institute of Standards and Technology and University of Colorado, Boulder, Colorado 80309-0440. Permanent \\ address: East China Normal University, Shanghai, China. Present address: International Bureau des Poids et Mesures, Sèvres, \\ France.
}

\begin{abstract}
We report a simple, robust 1-Hz linewidth laser system enabled by an improved mounting strategy for optical reference cavities used for laser frequency stabilization.
\end{abstract}

(C) 2005 Optical Society of America

OCIS codes: $120.3930,140.3530$

\section{Introduction}

We are constructing a sub-Hertz linewidth laser which will interrogate the attractive optical clock transitionof ${ }^{1} \mathrm{~S}_{0}-{ }^{3} \mathrm{P}_{0}$ in $\mathrm{Sr}$ at $698 \mathrm{~nm}$. As is common [1-3], the laser is pre-stabilized to a high finesse, highly stable Fabry-Perot cavity. The resulting laser stability at Fourier frequencies above $\sim 5 \mathrm{~Hz}$ is usually determined by the stability of the physical dimension of the Fabry-Perot frequency reference: at lower Fourier frequencies contributions also come from the fidelity of the laser frequency error signal and the inadvertent coupling of the cavity to external optical components by optical feedback [4], and ultimately at longer times, thermal variations and material creep will dominate. Considering the frequency range 1-100 $\mathrm{Hz}$, the dominant environmental noise is seismic acceleration, which leads to elastic deformation of the frequency reference cavity. Vibration isolation is required [1], with the needed extent being dependent on the environment and acceleration sensitivity of the cavity in relation to the objective noise level. Our approach has been to minimize the acceleration sensitivity of the cavity by mounting a short cavity [5] symmetrically about the center of mass (for a cavity of symmetrical shape) [6]. For an axial acceleration, this leads to (almost) equal amounts of compression and elongation in the two halves, that is some common mode rejection of the change in length with acceleration. Vertical mounting makes definition of the symmetry plane more apparent and convenient.

\section{Experiment}

The construction of the $698 \mathrm{~nm}$ system is in progress and awaits testing. Development experiments, described here, were done at $1064 \mathrm{~nm}$ where components were readily available. A ULE Fabry-Perot cavity of length $50 \mathrm{~mm}$ and diameter $12.7 \mathrm{~mm}$ has a linewidth of $65 \mathrm{kHz}$ at $1064 \mathrm{~nm}$ and was mounted in a center-drilled zerodur disc. The face of the disc had three counterbored holes drilled through to the midplane, with a corresponding set drilled from the other side to maintain the center of mass in the midplane. The cavity was attached to the disc with silicone RTV, aligning the center of masses of the cavity and disc within $\sim 0.5 \mathrm{~mm}$ : silicone being used to mitigate the stresses of the three-point mounting in the disc. This structure rested vertically on three aluminum posts, rigidly connected to a vacuum can. Under each of three support legs of the can, a piezoelectric actuator was used to displace the structure, and thus allow measurement of a transfer function of cavity deformation as a function of cavity acceleration. Cavity deformation was measured by measuring the frequency excursion of an Nd:YAG laser locked this cavity [7] in a heterodyne beat with a second, independently stabilized laser. In 


\section{CWJ5}

2005 Conference on Lasers \& Electro-Optics (CLEO)

repeated sweeps, mass was added to the end of the cavity to move the center of mass about the support point and reduce the acceleration sensitivity of the cavity. The orthogonality of the drive limited the reduction of the acceleration sensitivity of the cavity to $20 \mathrm{kHz} / \mathrm{ms}^{-2}$ : the added mass was $\sim 0.7 \mathrm{~g}$. Without the common mode rejection, the acceleration sensitivity would be $>2 \mathrm{MHz} / \mathrm{ms}^{-2}$.

The can housing the cavity was temperature controlled and mounted on a vibration isolation platform (Minus K). The laser was again servo-locked to the cavity and compared in a heterodyne beat with a second, independent system: the fiber used for the light transfer employing a Doppler noise cancellation system [8]. The beat was mixed down to $50 \mathrm{kHz}$ with a radio frequency source that often had a linear chirp of $\sim 20 \mathrm{~Hz} / \mathrm{s}$ to take out small drifts thought to be thermal in origin. This signal was examined on a Fast Fourier Transform analyzer and the result is plotted in figure 1.

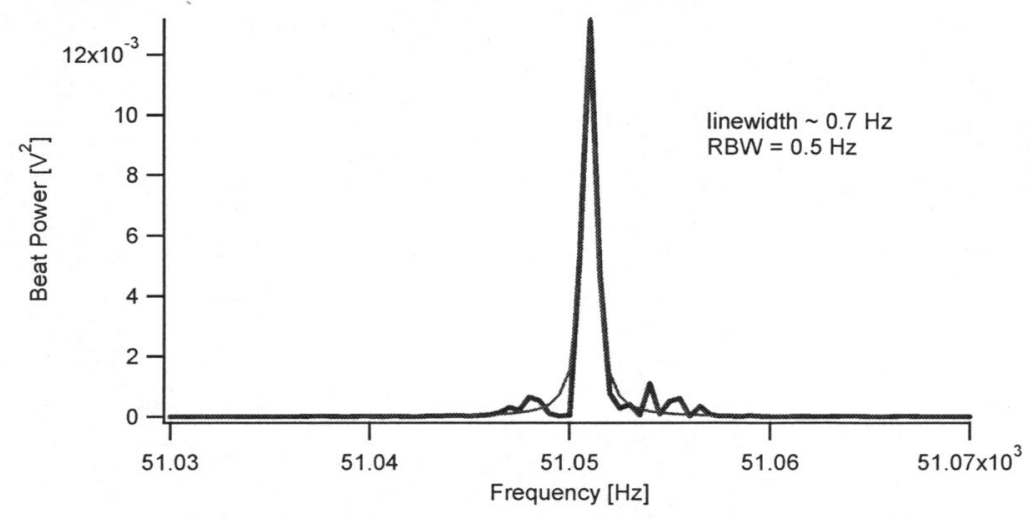

Fig. 1. Heterodyne beat between two independently stabilized lasers

As a diagnostic, a linear frequency noise density spectrum of the beat was generated using a frequency to voltage converter. This shows a generally flat noise floor which corresponds closely with the shot noise floor of the discrimination system. The peaks at $85 \mathrm{~Hz}$ are the feedthrough of seismic acceleration at an internal mechanical mode of the vibration isolation platform. The low frequency $1 / \mathrm{f}$ floor is thought to be due to thermal drifts of the cavity and possibly the expansion of the vacuum can stressing the cavity.

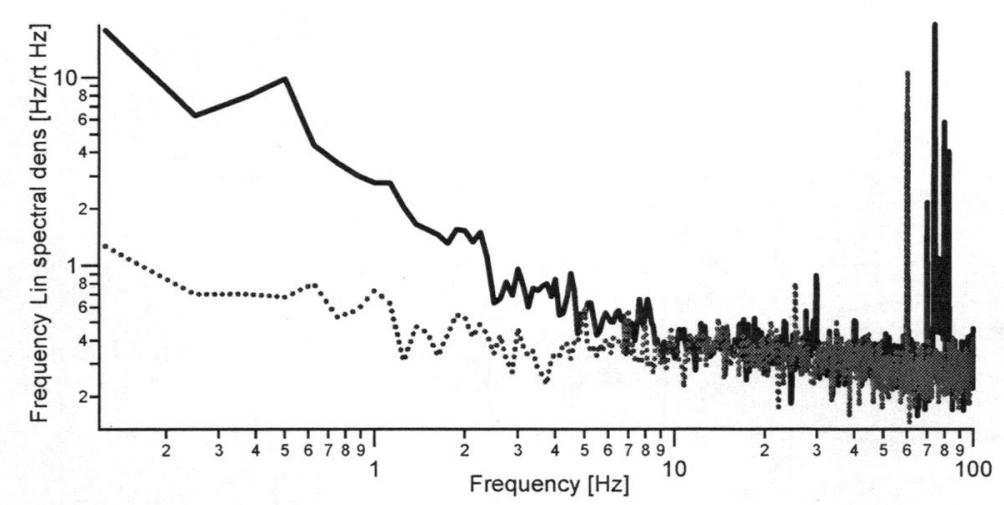

Fig. 2. The solid line is the frequency noise spectral density of the beat. The dashed line is the noise floor of the worse of the two lasers' error signal plotted in frequency units

In summary, we have developed a mounting for a Fabry-Perot cavity which provides common mode rejection of the elastic deformation of accelerations. This allows a commonly available and compact 


\section{CWJ5}

2005 Conference on Lasers \& Electro-Optics (CLEO)

vibration isolation system to be used to attenuate seismic noise sufficiently that the resulting linewidth of a frequency stabilized laser is at the $\mathrm{Hz}$ level.

\section{References}

1 B. C. Young, F. C. Cruz, W. M. Itano, and J. C. Bergquist, "Visible lasers with subhertz linewidths," Phys. Rev. Lett., 82, 37993802 (1999).

2 S. A. Webster, Oxborrow, Mark, and Gill, Patrick, " Subhertz-linewidth Nd:YAG laser," Opt. Lett., 29, 1497-1499 (2004).

3 A. Y. Nevsky, M. Eichenseer, J. von Zanthier, and H. Walther, "A Nd : YAG Laser with short-term frequency stability at the Hertzlevel," Optics Communications, 210, 91-100 (2002).

4 C. Salomon, D. Hils, and J. L. Hall, "Laser stabilization at the milliHertz level," JOSA B, 5, 1576-87 (1988).

5 M. Zhu and J. L. Hall, "Frequency stabilization of tunable lasers," in Atomic, Molecular and Optical Physics: Electromagnetic Radiation, Experimental Methods in the Physical Sciences Series Vol. 29C, F. B. Dunning and R. G. Hulet, ed. (Academic, San Diego, 1997).

6 M. Notcutt, C. T. Taylor, A. G. Mann, R. Gummer, and D. G. Blair, "Cryogenic system for a sapphire Fabry-Perot optical frequency standard," Cryogenics, 36, 13-16 (1996).

7 R. W. P. Drever, J. L. Hall, F. V. Kowalski, J. Hough, G. M. Ford, A. J. Munley, and H. Ward, "Laser phase and frequency stabilization using an optical resonator," Applied Physics, B31, 97-105 (1983).

8 L.-S. Ma, P. Jungner, J. Ye, and J. L. Hall, "Delivering the same optical frequency at two places: accurate cancellation of phase noise introduced by an optical fiber or other time-varying path," Opt. Lett., 19, 1777-9 (1994). 